\title{
RECURSIVE PSEUDO-WELL-ORDERINGS
}

\author{
BY \\ JOSEPH HARRISON( $\left.{ }^{1}\right)$
}

Introduction. This paper is devoted to a study of recursive linear orderings which have no hyperarithmetic descending sequences and hierarchies on these orderings. In the first section we discuss a method for generalizing certain results on recursive-well-orderings to such recursive pseudo-well-orderings. We prove that if $<_{R}$ is any such ordering, then transfinite induction holds on $<_{R}$ for $\Sigma_{1}^{1}$ formulae. This permits one to extend several other results for recursive wellorderings to such $<_{R}$. The possible order types of such relations is completely characterized by the result that, for some $\alpha<\omega_{1},<_{R}$ has order type $\omega_{1} \cdot(1+\eta)+\alpha$ where $\eta$ is the order type of the rationals in the open interval $(0,1)$.

In the second section we define a hierarchy on a recursive pseudo-well-ordering to be essentially a sequence of functions associated with each element of the field of $<_{R}$ and satisfying the same inductive conditions at successors and limits as the functions of the hyperarithmetic hierarchy. We obtain various results which show how the relation $<_{R}$ induces certain structures on the relations of recursive and hyperarithmetic reducibility between functions of the hierarchy. The most important of these is that if $\alpha_{a}$ and $\alpha_{b}$ are the functions associated with $a$ and $b$ in some hierarchy on $<_{R}$; and if $a<_{R} b$, and the segment between $a$ and $b$ is not well ordered, then everything hyperarithmetic in $\alpha_{a}$ is recursive $\alpha_{b}$. These facts can be applied to obtain a number of new results of interest in the study of hyperdegrees. These include the existence of pairs of hyperdegrees without a greatest lower bound; the existence, for a given hyperdegree, of an infinite descending sequence of hyperdegrees having the given one as a greatest lower bound; the existence of maximal densely ordered sets of hyperdegrees; the existence, for a given $\Sigma_{1}^{1}$ set $S$ containing a nonhyperarithmetic function, of a subset of the hyperdegrees of $S$ having the cardinality of the continuum and consisting of mutually incomparable hyperdegrees; the existence of a pair of hyperdegrees $[\alpha],[\beta]$ such that $0<[\alpha],[\beta]<0^{\prime}$ (the hyperdegree of Kleene's 0 ), with $[\alpha] \cap[\beta]=0$ and $[\alpha] \cup[\beta]=0^{\prime}$. In addition, our methods also yield the basic results on the existence of incomparable hyperdegree obtained in recent years via the methods of forcing and measure theory (see for example, Feferman [4], Spector [16], and Thomason [18]).

In the text much use is made of $O^{*}$, the set of notations for recursive linear orderings with no hyperarithmetic descending sequences which was introduced in

Received by the editors February 1, 1967.

( $\left.{ }^{1}\right)$ The author is a Postdoctoral Fellow in the Department of Preventive Medicine, Stanford University, Stanford, California. 
Feferman and Spector [5]. This is partly because it is convenient to have the function, "predecessor of", recursive on our orderings, but also because our work begins by solving some problems concerning $O^{*}$ that are implicit in Feferman [2].

The results reported in this paper were obtained while the author was a student of Professor Solomon Feferman at Stanford University. A more complete presentation of them has been given in the author's doctoral thesis [11]. Announcement of the results has also been made in [8], [9], and [10].

The author is indebted to Professor Feferman for his guidance during the research and preparation of this paper as well as for providing a new and fruitful notion (the predicate " $Q(\alpha, a)$ " of the second part). He is also indebted to Professor Joseph Schoenfield of Duke University who helped guide the research during 1964-1965 when Professor Feferman was on leave. He also had some helpful conversations with Professors Georg Kreisel and Dana Scott.

1. In this first section we derive some general properties of $O^{*}$. Some, although not all of these properties, are generalizations to $O^{*}$ of familiar properties of $O$. Let us recall the principal facts about $O^{*}$ from Feferman and Spector [5].

Definition. $O^{*}=\bigcap X\left(X \in H A \wedge\left[1 \in X \wedge z \in X \rightarrow 2^{z} \in X \wedge((\forall n)(\{e\}(n) \in X \wedge\{e\}(n)\right.\right.$ $\left.\left.\left.\prec\{e\}(n+1)) \rightarrow 3 \cdot 5^{e} \in X\right)\right]\right) . \prec$ is the recursively enumerable relation satisfying the conditions: (i) $1 \prec x$ if $x \neq 1$, (ii) $z<2^{z}$, (iii) $\{e\}(n) \prec 3 \cdot 5^{e}$, (iv) $a \prec b \wedge b \prec c \rightarrow$ $a \prec c$.

Fact 1. $O^{*}$ consists of integers $n$ for which $\{u: u \preccurlyeq n\}$ is well-ordered with respect to hyperarithmetic sequences and $\{u: u \preccurlyeq n\}$ only contains 1 , and $u$ of the form $2^{(u) 0}$, and $3 \cdot 5^{(u)_{2}}$ where $(\forall n)\left(\left\{(u)_{2}\right\}(n) \prec\left\{(u)_{2}\right\}(n+1)\right)$.

Fact 2. $O \varsubsetneqq O^{*}, O^{*} \in \Sigma_{1}^{1}$.

Fact 3. If $n \in O^{*}-O,\{u: u \preccurlyeq n\} \cap O$ is a $\Pi_{1}^{1}$ path through $O$. Conversely, if $P$ is a $\Pi_{1}^{1}$ path through $O$, then for some $n \in O^{*}-O,\{u: u \preccurlyeq n\} \cap O=P$.

Although one can attack problems about $O^{*}$ directly, it seems more natural to exploit the similarity in the definitions of $O$ and $O^{*}$ in the following way as suggested by Kreisel: since the definition of $O^{*}$ may be obtained from that of $O$ by restricting the function quantifier to range over the hyperarithmetic functions, the statement and proof of a result about $O$ can be translated into the statement and proof of a result about $O^{*}$ simply by relativizing the function quantifiers to the class of hyperarithmetic functions provided, of course, that all axioms and principles of proof remain valid in the class of hyperarithmetic functions. For example, a proof using only the $\Sigma_{1}^{1}$ axiom of choice would remain valid under this translation since the $\Sigma_{1}^{1}$ axiom of choice was verified to hold in the class of hyperarithmetic functions by Kreisel in [14].

We can also show that the stronger $\Sigma_{1}^{1}$ axiom of dependent choices is valid in the class of hyperarithmetic functions. This axiom follows from: if

$$
(\forall \alpha)(\exists \beta)(\forall x) R(\bar{\alpha}(x), \bar{\beta}(x))
$$


where $R$ is recursive, then

$$
(\forall \eta)(\exists \alpha)(\forall n)(\forall x)(\alpha(1, x)=\eta(x) \wedge R(\bar{\alpha}(n, x), \bar{\alpha}(n+1, x))) .
$$

To verify this axiom for the class of hyperarithmetic functions we prove first the following theorem.

THEOREM 1.1. Suppose $X, K \in \Pi_{1}^{1}, X \neq \phi$, and $(\forall x)(x \in X \rightarrow(\exists y)(y \in X \wedge K(x, y)))$ then

$$
(\forall x)\left(x \in X \rightarrow(\exists \alpha)_{H A}(\alpha(0)=x \wedge(\forall n)(\alpha(n) \in X \wedge K(\alpha(n), \alpha(n+1)))) .\right.
$$

For the proof we need the following lemma whose proof is adapted from Kreisel [14].

Lemma (UNiformization TheOREM For $\Pi_{1}^{1}$ Relations). If $P(x, y)$ is $\Pi_{1}^{1}$ then there exists $P^{\prime}(x, y), \Pi_{1}^{1}$ such that

(a) $P^{\prime}(x, y) \rightarrow P(x, y)$,

(b) $(\forall x)\left((\exists y) P(x, y) \rightarrow(\exists y) P^{\prime}(x, y)\right)$.

Proof. Since $P(x, y)$ is $\Pi_{1}^{1}$, there exists $R$ primitive recursive such that $P(x, y)$ $\leftrightarrow(\forall \alpha)(\exists z) R(\bar{\alpha}(z), x, y)$. Following Kleene [12], $(\forall \alpha)(\exists z) R(\bar{\alpha}(z), x, y) \leftrightarrow$ the unsecured sequences of $R(\bar{\alpha}(z), x, y)$ are well ordered. Let $P^{\prime}(x, y) \leftrightarrow P(x, y)$ $\wedge(\forall u)(\forall \alpha)$ ( $\alpha$ is not an isomorphism of the unsecured sequences of $R(\bar{\alpha}(z), x, u)$ onto a proper initial segment of those of $R(\bar{\alpha}(z), x, y)) \wedge(\forall w)(w<y \rightarrow(\forall \beta)(\beta$ is not an isomorphism of the unsecured sequences of $R(\bar{\alpha}(z), x, w)$ into those of $R(\bar{\alpha}(z), x, y)))$.

It is easy to see that $P^{\prime}(x, y)$ has the properties (a) and (b).

Proof of theorem. Let $P(x, y)$ be the predicate $K(x, y) \wedge y \in X$. Then $P(x, y) \in \Pi_{1}^{1}$ and by hypothesis $(\forall x)(x \in X \rightarrow(\exists y) P(x, y))$. Choose $P^{\prime}(x, y)$ as in the lemma. Then $(\forall x)\left(x \in X \rightarrow(\exists y) P^{\prime}(x, y)\right)$ and $P^{\prime}(x, y) \rightarrow K(x, y) \wedge y \in X$. Define $\alpha$ as follows:

$$
\alpha(0)=a, \quad \alpha(x+1)=\iota y P^{\prime}(\alpha(x), y)
$$

is always defined and $\alpha \in \Pi_{1}^{1}$ since

$$
\begin{aligned}
\alpha(x)=y \leftrightarrow(\exists s)(\operatorname{Seq}(s) & \wedge \operatorname{Lh}(s)=x+1 \wedge(i)(i<\operatorname{Lh}(s) \\
& \left.\left.\rightarrow\left(i=0 \wedge(s)_{i}=a \vee i \neq 0 \wedge P^{\prime}\left((s)_{i-1},(s)_{i}\right)\right)\right)\right) .
\end{aligned}
$$

Hence also $\alpha \in H A$, so $\alpha$ has the required properties.

Now we can show

THEOREM 1.2. ( $\Sigma_{1}^{1}$ AXIOM OF DEPENDENT CHOICES FOR HYPERARITHMETIC FUNCTIONS). If $(\forall \alpha)_{H A}(\exists \beta)_{H A}(\forall x) R(\bar{\alpha}(x), \bar{\beta}(x))$, where $R$ is recursive, then

$$
(\forall v)_{H A}(\exists \alpha)_{H A}(\forall x)(\forall n)(R(\bar{\alpha}(n, x), \bar{\alpha}(n+1, x)) \wedge \alpha(1, x)=\nu(x)) .
$$

Proof. Let $X=\left\{2^{e} \cdot 3^{y}: y \in O \wedge\{e\}^{H_{y}}\right.$ is total $\}$. Let $K(u, v)$ be the predicate

$$
(\forall x) R\left(\left\{(u)_{0}\right\}^{H_{(u)}}(x),\left\{(v)_{0}\right\}^{H_{(v)}}(x)\right) .
$$


Using the standard techniques of Kleene, we see that $X, K \in \Pi_{1}^{1}$, and the hypothesis of the theorem implies $(\forall u)(u \in X \rightarrow(\exists v)(v \in X \wedge K(u, v))$.

Let $\nu \in H A$. Then for some $2^{p} \cdot 3^{q}$ in $X, \nu=\{p\}^{H_{q}}$. By Theorem 1.1, there is a hyperarithmetic $f$ such that $\left.f(0)=2^{p} \cdot 3^{q},(\forall n) K(f(n), f(n+1)) \wedge f(n) \in X\right)$. Let

$$
\alpha(n, x)=\left\{(f(n))_{0}\right\}^{H_{(f(n))}}{ }_{1}(x) .
$$

Then $\alpha$ is hyperarithmetic and satisfies the conclusion of Theorem 1.2.

Kreisel observed that several of the results which we had obtained earlier about $O^{*}$, in particular Theorems 1.3,1.4, and 1.5 below, could be subsumed under the general principle discussed above by verifying that the proofs of the corresponding results about $O$ made use at most of the $\Sigma_{1}^{1}$ axiom of dependent choices. Three such results are as follows: first, the $\Pi_{1}^{1}$ completeness of $O$ which requires only the arithmetic comprehension axiom. Second is the uniqueness of any isomorphism between an initial segment of $\{y: y \preccurlyeq a\}$ and an initial segment of $\{y: y \preccurlyeq b\}$ for $a, b \in O$. The proof of this also requires only the arithmetic comprehension axiom. Third is the least element principle for $\Sigma_{1}^{1}$ subsets of $\{y: y \preccurlyeq a\}$ where $a \in O$. This can be proved from the definition of $O$ using the $\Sigma_{1}^{1}$ axiom of dependent choices as follows: suppose $X=\{n:(\exists \beta)(\forall u) R(n, \bar{\beta}(u))\}$ where $R$ is recursive. $X \subset\{y: y \preccurlyeq a\}$, and $(\forall x)(x \in X \rightarrow(\exists y)(y \in X \wedge y \prec x)$. We will show $a \notin O$. By assumption

$$
\begin{gathered}
(\forall x)(\forall \alpha)((\forall u) R(x, \bar{\alpha}(u)) \rightarrow(\exists \beta)(\exists y)(\forall u) R(y, \bar{\beta}(u)) \wedge y \prec x), \\
(\forall \alpha)(\exists \beta)((R(\alpha(0), \overline{\lambda y \alpha(y+1)}(u)) \rightarrow((\forall u) R(\beta(0), \overline{\lambda y \beta(y+1)}(u)) \wedge \beta(0) \prec \alpha(0)) .
\end{gathered}
$$

Choose $\alpha$ so that $(\forall u) R(\alpha(0), \overline{\lambda y \alpha(y+1)}(u))$. By the $\Sigma_{1}^{1}$ axiom of dependent choices,

$$
\begin{gathered}
(\exists \nu)((\forall n)((\forall u) R(\nu(n, 0), \overline{\lambda y \nu(n, y+1)}(u)) \rightarrow((\forall u) R(\nu(n+1,0), \overline{\lambda y \nu(n+1, y+1)}(u)) \\
\wedge v(n+1,0) \prec v(n, 0))) \wedge(\forall u)(\nu(1, u)=\alpha(u)) .
\end{gathered}
$$

Hence $\nu(1,0)=\alpha(0) \preccurlyeq a$ by choice of $\alpha$, and $(\forall n)(\nu(n+1,0) \prec \nu(n, 0))$. So $a \notin O$.

It follows from our earlier remarks that these results remain valid when relativized to the class of hyperarithmetic functions. The relativization of the first is

THEOREM 1.3. Suppose $R(\bar{\alpha}(x), n)$ is recursive. Let $\phi$ be the function defined in Kleene [12] which reduces $(\forall \alpha)(\exists x) R(\bar{\alpha}(x), n)$ to $O$. Then

$$
(\forall \alpha)_{H A}(\exists x) R(\bar{\alpha}(x), n) \leftrightarrow \phi(n) \in O^{*} .
$$

\section{Hence $O^{*}$ is $\Sigma_{1}^{1}$ complete.}

The last remark follows by Spector's Theorem [15] which shows that the class of predicates of the form $(\forall \alpha)_{H A}(\exists x) R(\bar{\alpha}(x), n)$, where $R$ is recursive, is just the class of $\Sigma_{1}^{1}$ predicates. Theorem 1.3 was first obtained by Feferman [3]. The relativization of the second result is

THEOREM 1.4. If $a, b \in O^{*}$ then any hyperarithmetic isomorphism between an initial segment of $\{y: y \preccurlyeq a\}$ and an initial segment of $\{y: y \preccurlyeq b\}$ is unique. 
It follows immediately from this theorem that if $\{y: y \preccurlyeq a\}$ is hyperarithmetically isomorphic to an initial segment of $\{y: y \preccurlyeq b\}$ and vice versa, then the composition of the two maps must be the identity, and both maps are isomorphisms onto.

By Spector's Theorem, the class of sets expressible in the form

$$
\left\{n:(\exists \alpha)_{H A}(\forall x) R(\bar{\alpha}(x), n)\right\}
$$

where $R$ is recursive, is just the class of $\Pi_{1}^{1}$ sets. By relativizing the third result and applying this fact we obtain

THEOREM 1.5. Suppose $a \in 0^{*} X \in \Pi_{1}^{1}, X \subseteq\{y: y \preccurlyeq a\}, X \neq \phi$. Then $X$ has a least element with respect to $\preccurlyeq$.

Corollary 1.6. (i) Suppose $a \in O^{*}, X \subseteq\{y: y \preccurlyeq a\}, X \in \Sigma_{1}^{1}$ and $X$ is inductive, i.e., $(\forall y)(y \preccurlyeq z \rightarrow y \in X) \rightarrow z \in X$ provided $z \preccurlyeq a$. Then $X=\{y: y \preccurlyeq a\}$. (ii) $O^{*}=$ $\cap X\left(X \in \Sigma_{1}^{1} \wedge\left[1 \in X \wedge z \in X \rightarrow 2^{z} \in X \wedge((\forall n)(\{e\}(n) \in X \wedge\{e\}(n) \prec\{e\}(n+1))\right.\right.$ $\left.\left.\left.\rightarrow 3.5^{e} \in X\right)\right]\right)$.

Proof. (i) is immediate from the theorem. Feferman and Spector prove

$$
\begin{aligned}
O^{*}=\bigcap X(X \in H A \wedge[1 \in X \wedge z \in X & \rightarrow 2^{z} \in X \wedge((\forall n)(\{e\}(n) \in X \\
& \left.\left.\left.\wedge\{e\}(n) \prec\{e\}(n+1)) \rightarrow 3 \cdot 5^{e} \in X\right)\right]\right) .
\end{aligned}
$$

Hence $O^{*}$ includes the intersection given in (ii). Suppose $X \in \Sigma_{1}^{1}$ has the closure property given in square brackets and $a \in O^{*}$. We want to show $a \in X$. Let $Z=\{y: y \preccurlyeq a\} \cap X$. It follows from the bracketed condition that $Z$ is inductive and clearly $Z \in \Sigma_{1}^{1}$ if $X \in \Sigma_{1}^{1}$. Hence $Z=\{y: y \preccurlyeq a\}$ and $a \in X$. So $O^{*} \subseteq X$ and $O^{*}$ is included in the intersection given in (ii). Having proven both inclusions, we have proven (ii).

COROllary 1.7. All theorems on $+_{o}{ }_{0} o$ in Kleene [12] continue to hold when $O$ is replaced by $O^{*}$. (These theorems include the closure of $O^{*}$ under notation arithmetic and the basic properties of these operations.)

Proof. All of these theorems are proved by induction on a predicate of the form $\phi(b) \in O$, or $R(b)$ where $\phi$ and $R$ are hyperarithmetic. Any proof involving such $R(b)$ also works for $O^{*}$. If we replace the predicate " $\phi(b) \in O$ " by " $\phi(b) \in O$ *", we obtain a $\Sigma_{1}^{1}$ predicate and the inductive arguments remain valid. Closure of $O$ under $t_{o}$ and ${ }_{0} o$ was first proved by Feferman in [2] using rather elaborate arguments. They are now superseded by the foregoing.

The reverse side of the coin is that, in the case of a result about $O$ which does not relativize to $O^{*}$, we have the corollary that it could not have been proven solely by means of the $\Sigma_{1}^{1}$ axiom of dependent choices.

As an example, consider the least element principle for $\Pi_{1}^{1}$ subsets of $\{y: y \preccurlyeq a\}$ where $a \in O$. Its relativization to the class of hyperarithmetic functions is the least element principle for $\Pi_{1}^{1(H A)}=\Sigma_{1}^{1}$ subsets of $\{y: y \preccurlyeq a\}$ where $a \in O^{*}$. This is false 
since if $a \in O^{*}-O,\{y: y \preccurlyeq a\} \cap O^{*}-O$ is a $\Sigma_{1}^{1}$ subset of $\{y: y \preccurlyeq a\}$ with no least element.

We now turn to an analysis of the order type of $\{y: y \preccurlyeq a\}$ where $a \in O^{*}-O$. In [2] Feferman proved that, for $a \in O^{*}$, one could define a function $P(a)$ having the essential properties of ordinal exponentiation $2^{<}$and such that if $a \in O^{*}-O$, then $\{y: y \preccurlyeq P(a)\}$ has a subset which is densely ordered. The proof is based on the fact that if $<$ is any linear ordering which is not a well-ordering, $2^{<}$contains a subset which is densely ordered.

Proceeding along somewhat different lines, we have obtained the following more general and informative theorem.

THEOREM 1.8. Suppose $a \in O^{*}-O$. Let $1+\eta$ be the order type of the rationals in $[0,1)$. Then there exists a unique $\alpha<\omega_{1}$ such that $\{y: y \preccurlyeq a\}$ has order type $\omega_{1} \cdot(1+\eta)+\alpha$. (Here "." and "+" denote the product and sum of order types.)

Proof. If $y_{1}, y_{2} \preccurlyeq a$ say $y_{1} \sim y_{2}$ iff the segment determined by $\preccurlyeq$ between $y_{1}$ and $y_{2}$ is well ordered. $\sim$ is clearly an equivalence relation. Moreover, the equivalence classes are segments since $y_{1} \sim y_{2}, y_{1} \preccurlyeq y_{3} \preccurlyeq y_{2}$ clearly implies $y_{1} \sim y_{3} \sim y_{2}$. Let $E(y)$ be the equivalence class determined by $y$. Then

$$
\begin{aligned}
E(y)=\{z: z \preccurlyeq a \wedge(\forall \alpha)(((\forall i)(z \preccurlyeq \alpha(i) \preccurlyeq y) & \vee(\forall i)(y \preccurlyeq \alpha(i) \preccurlyeq z)) \\
& \rightarrow(\exists x)(\alpha(x+1) \nless \alpha(x)))\} .
\end{aligned}
$$

So $E(y)$ is $\Pi_{1}^{1}$ and has a least element by Theorem 1.5. $E(a)$ is clearly the last equivalence class. Let $b$ be its first element. Then $E(a)=\{y: b \preccurlyeq y \preccurlyeq a\}$ is recursively enumerable and is well ordered by the recursively enumerable relation $\preccurlyeq$. Hence $E(a)$ has order type $\alpha<\omega_{1}$.

The statement of the theorem will follow if we can show (a) each equivalence class except the last has order type $\omega_{1}$; (b) between any two equivalence classes there is a third. The proof of (a) is exactly like the proof in [5] of the first part of Fact 3 given above.

Proof of (b). Suppose $E\left(y_{0}\right)$ and $E\left(y_{1}\right)$ are distinct equivalence classes, $y_{0} \prec y_{1}$, and $y_{1}$ is the first element of its equivalence class. Note that if $y \preccurlyeq a$ then $E(y)$ is inductive. Hence if for all $u, y_{0} \preccurlyeq u \prec y_{1}, u \in E\left(y_{0}\right)$, then $y_{1} \in E\left(y_{0}\right)$ which contradicts the choice of $y_{0}$ and $y_{1}$. Hence there exists $y_{2}, y_{0} \prec y_{2} \prec y_{1}$, such that $y_{2} \notin E\left(y_{0}\right)$. Since $y_{1}$ is the first element of its equivalence class we also have $y_{2} \notin E\left(y_{1}\right)$. Thus $E\left(y_{2}\right)$ is a distinct equivalence class between $E\left(y_{0}\right)$ and $E\left(y_{1}\right)$.

It follows from Theorem 1.8 that if $a, b \in O^{*}-O,\{y: y \preccurlyeq a\}$ is isomorphic to an initial segment of $\{y: y \preccurlyeq b\}$ and conversely. It is natural to ask whether one of these isomorphisms can be chosen to be hyperarithmetic. This is equivalent to asking whether the following theorem about $O$ relativizes to $O^{*}: a, b \in O \rightarrow(\exists \alpha)$ ( $\alpha$ is an isomorphism of $\{y: y \preccurlyeq a\}$ onto an initial segment of $\{y: y \preccurlyeq b\}$ or conversely). The answer is negative. In [13] Kreisel proves the existence of two recursive linear orderings without hyperarithmetic descending sequences which 
are not comparable in this sense by a hyperarithmetic function. One can show that the Markwald-Spector reduction of $W$ to $O$ also reduces $W^{*}$ to $O^{*}$, and one can use this reduction to obtain hyperarithmetically incomparable elements of $O^{*}$ Actually one can prove a mildly stronger result directly.

TheOREM 1.9. Suppose $a \in O^{*}-O, O \subseteq S \subseteq O^{*}, S \in \Sigma_{1}^{1}$. There exists $b \in S$ such that $\{y: y \preccurlyeq a\}$ is not hyperarithmetically isomorphic to an initial segment of $\{y: y \preccurlyeq b\}$ and conversely.

Proof. We need two lemmas.

Lemma 1. Suppose $(\forall y)(\exists \alpha)_{H A}(\forall x) P(y, \bar{\alpha}(x))$ where $P \in \Pi_{1}^{1}$. Then

$$
(\exists \alpha)_{H A}(\forall y)(\forall x) P(y, \bar{\alpha}(y, x)) .
$$

Proof. One writes $P(y, \bar{\alpha}(x))$ in the form $(\exists \nu)_{H A}(\forall u) R(y, \bar{\alpha}(x), \bar{\nu}(u))$ and applies the $\Sigma_{1}^{1}$ axiom of choice for hyperarithmetic functions.

Lemma 2. If $y \in O$, then there exist $z_{0}, z_{1} \in O$ depending on $y$, such that $\left|z_{0}\right|=\left|z_{1}\right|$ but $\left\{u: u \preccurlyeq z_{0}\right\}$ and $\left\{u: u \preccurlyeq z_{1}\right\}$ are not isomorphic by any function recursive in $H_{y}$.

Proof. If not, then for some $y \in O$, if $u, v \in O$ and $|u|=|v|$ then $\{z: z \preccurlyeq u\}$ and $\{z: z \preccurlyeq v\}$ are isomorphic by a function recursive in $H_{y}$. Hence for $u \in O, n \in O_{|u|}$ $\leftrightarrow\left(\{z: z \preccurlyeq n\}\right.$ is linearly ordered $\wedge(\exists \alpha)$ ( $\alpha$ is recursive in $H_{y} \wedge \alpha$ is an isomorphism of $\{z: z \preccurlyeq n\}$ onto an initial segment of $\{z: z \preccurlyeq u\})$ ). So $u \in O$ implies that $O_{|u|}$ is arithmetic in $H_{y}$. This contradicts the results of Spector [17].

Proof of Theorem 1.9. Suppose the theorem is false. Then $(\forall b)\left(b \in S \rightarrow(\exists \alpha)_{H A}\right.$ ( $(\alpha$ maps $\{y: y \preccurlyeq b\}$ isomorphically onto an initial segment of $\{y: y \preccurlyeq a\})$ or ( $\alpha$ maps $\{y: y \preccurlyeq a\}$ isomorphically onto an initial segment of $\{y: y \preccurlyeq b\}))$ ). By standard manipulations of quantifiers using the fact $S \in \Sigma_{1}^{1}$ this can be put in the form

$$
(\forall b)(\exists \alpha)_{H A}(\forall x) P(b, a, \bar{\alpha}(x)),
$$

where $P$ is $\Pi_{1}^{1}$. Hence by Lemma 1 ,

$$
(\exists \alpha)_{H A}(\forall b)(\forall x) P(b, a, \bar{\alpha}(b, x)) .
$$

Reversing the quantifier manipulations, we find $(\exists \alpha)_{H A}(\forall b)(b \in S \rightarrow \lambda x \alpha(b, x)$ is an isomorphism of $\{y: y \preccurlyeq a\}$ onto an initial segment of $\{y: y \preccurlyeq b\}$ or it is an isomorphism of $\{y: y \preccurlyeq b\}$ onto an initial segment of $\{y: y \preccurlyeq a\})$. Let $\alpha \in H A$ have this property. Then for $b \in O \subseteq S$, we must have that $\lambda x \alpha(b, x)$ is an isomorphism of $\{y: y \preccurlyeq b\}$ onto an initial segment of $\{y: y \preccurlyeq a\}$. Let $\alpha_{b}(x)=\alpha(b, x)$. Then if $b_{1}, b_{2} \in O$, and $\left|b_{1}\right|=\left|b_{2}\right|, \alpha_{b_{1}}^{-1} \cdot \alpha_{b_{2}}$ is an isomorphism between $\left\{y: y \preccurlyeq b_{1}\right\}$ and $\left\{y: y \preccurlyeq b_{2}\right\}$ recursive in $\alpha$ and hence in some fixed $H_{y}$. This contradicts Lemma 2, so the theorem is established.

COROLlaRY 1.10. The following statement is not provable solely by means of the $\Sigma_{1}^{1}$ axiom of dependent choices: $a, b \in O \rightarrow(\exists \alpha)$ ( $\alpha$ is an isomorphism of $\{y: y \preccurlyeq a\}$ onto an initial segment of $\{y: y \preccurlyeq b\}$ or vice versa). 
We conclude this section with an interesting open question about $O^{*}$. Given $P_{1}, P_{2}, \Pi_{1}^{1}$ paths through $O$, does there exist a total hyperarithmetic function $f$ which maps $P_{1}$ isomorphically on $P_{2}$ ? Let $a_{1}$ and $a_{2} \in O^{*}-O$ be chosen so that $\left\{y: y \preccurlyeq a_{1}\right\} \cap O=P_{1},\left\{y: y \preccurlyeq a_{2}\right\} \cap O=P_{2}$. If $f$ has the required property and $S=\left\{y: y \preccurlyeq a_{1} \wedge f\right.$ is an isomorphism of $\{z: z \preccurlyeq y\}$ onto $\left.\{z: z \preccurlyeq f(y)\}\right\}$, then $S$ is hyperarithmetic and $S \supseteq P_{1} . S \neq P_{1}$ since $P_{1}$ is not hyperarithmetic. Hence there exists $y \preccurlyeq a_{1}, y \notin O$ such that $\{z: z \preccurlyeq y\}$ and $\{z: z \preccurlyeq f(y)\}$ are isomorphic by $f$. Conversely, if there exist $b_{1}, b_{2} \in O^{*}-O, b_{1} \preccurlyeq a_{1}, b_{2} \preccurlyeq a_{2}$ such that $\left\{z: z \preccurlyeq b_{1}\right\}$ and $\left\{z: z \preccurlyeq b_{2}\right\}$ are hyperarithmetically isomorphic, so are $P_{1}$ and $P_{2}$.

Hence, there exist $\Pi_{1}^{1}$ paths through $O$ which are not hyperarithmetically isomorphic iff there exist $a, b \in O^{*}-O$ such that for all $a_{0} \preccurlyeq a, b_{0} \preccurlyeq b, a_{0}, b_{0} \in O^{*}-O$, $\left\{z: z \preccurlyeq a_{0}\right\}$ and $\left\{z: z \preccurlyeq b_{0}\right\}$ are not hyperarithmetically isomorphic. This last condition is prima facie stronger than saying that $\{z: z \preccurlyeq a\}$ is not hyperarithmetically isomorphic to an initial segment of $\{z: z \preccurlyeq b\}$ and conversely $\left({ }^{2}\right)$.

2. In this section we investigate hierarchies on elements of $O^{*}$. By a hierarchy for $a \in O^{*}$ we mean a function of two variables $\alpha(y, z)$ which satisfies the following arithmetic condition:

$$
\begin{array}{r}
(\forall y)(\forall z)\left(( y \npreceq a \rightarrow \alpha ( y , z ) = 1 ) \wedge \left(y=2^{(y)_{0}} \wedge y \preccurlyeq a \rightarrow(\alpha(y, z)=0\right.\right. \\
\leftrightarrow(\exists w) T_{1}^{1}\left(\bar{\alpha}\left((y)_{0}, w\right), z, z, w\right) \wedge\left(y=3 \cdot 5^{(y)_{2}} \wedge y \preccurlyeq a \rightarrow \alpha(y, z)\right. \\
\left.\left.=\alpha\left(\left\{(y)_{2}\right\}\left((z)_{0}\right),(z)_{1}\right)\right)\right) .
\end{array}
$$

We abbreviate this condition " $H(\alpha, a)$ ". If $H(\alpha, a)$, let $\alpha_{y}=\lambda z \alpha(y, z)$. Then the sequence $\left\{\alpha_{y}\right\}_{y \prec a}$ has the property that if $y=2^{(y)_{0}} \preccurlyeq a$, then $\alpha_{y}=\left(\alpha_{(y)_{0}}\right)^{\prime}$. If $y=3 \cdot 5^{(y)_{2}}$ $\preccurlyeq a$, then

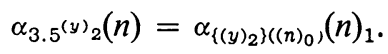

Conversely, given a sequence $\left\{\alpha_{y}\right\}_{y \prec a}$ with these properties, define $\alpha$ by $\alpha(y, z)=\alpha_{y}(z)$ if $y \preccurlyeq a, \alpha(y, z)=1$ otherwise. Then $H(\alpha, a)$.

Hierarchies with $\alpha_{1}=1$ were first implicitly used in Gandy's proof of Spector's Theorem in [7]. This can be expressed in terms of $H$ roughly as follows: let $a \in O^{*}-O$ be fixed. Then $n \in O \leftrightarrow(\exists \alpha)_{H A}(\exists \beta)_{H A} H(\alpha, n) \wedge \alpha_{1}=1 \wedge \beta$ is an isomorphism of $\{y: y \preccurlyeq n\}$ onto an initial segment of $(\{y: y \preccurlyeq a\}) \wedge A(n)$, where $A(n)$ is arithmetic. No other applications of such hierarchies seem to have been known. We began a systematic study of hierarchies on $a \in O^{*}$ with work that was announced in [8] and [9]. During that period Feferman realized that hierarchies with $\alpha_{1}=1$ satisfying a certain predicate $Q(\alpha, a)$ involving a strong additional inductive condition could be used to prove the independence of the $\Sigma_{1}^{1}$ axiom of choice from the hyperarithmetic comprehension axiom. This result was announced in [1]. We were then able to use the predicate $Q$ to strengthen certain of our earlier results (notably Theorems 2.6, 2.8, and 2.9 below) and to obtain a new result about

$\left({ }^{2}\right)$ The author has learned recently that R. M. Solovay has proven the existence of $\Pi_{1}^{1}$ paths through $O$ which are not hyperarithmetically isomorphic. 
hyperarithmetic reducibility (Theorem 2.11 below). We modify the definition of $Q(\alpha, a)$ given in [1] slightly by now allowing $\alpha_{1}$ to be arbitrary, i.e., we take $Q(\alpha, a) \leftrightarrow H(\alpha, a) \wedge(\forall \beta)(\beta$ is hyperarithmetic in $\alpha \wedge \beta \subseteq\{y: y \preccurlyeq a\}$ then $\beta$ has a least element). The basic existence theorem for this predicate is now given by the following theorem. As we shall see later, essentially new considerations beyond the axiomatic ones discussed in section one are needed here.

TheOREM 2.1. Suppose $a \in O^{*}-O, \omega_{1}^{\nu}=\omega_{1}$. Then there exists $b \preccurlyeq a, b \in O^{*}-O$ and $\alpha$ such that $Q(\alpha, b) \wedge \alpha_{1}=\nu$.

Proof. Let $S_{a, v}=\left\{b: b \preccurlyeq a \wedge(\exists \alpha)\left(Q(\alpha, b) \wedge \alpha_{1}=\nu\right)\right\} . \quad S_{a, v} \in \Sigma_{1}^{1, \nu} \quad$ and $S_{a, \nu} \supset O$ $\cap\{y: y \preccurlyeq a\}=P$ which is a $\Pi_{1}^{1}$ path through $O . P \in \Pi_{1}^{1}$, therefore, $P \in \Pi_{1}^{1}, v$. Since $\omega_{1}^{P}>\omega_{1}$, but $\omega_{1}^{v}=\omega_{1}^{\nu}$, it follows that $P \notin \Sigma_{1}^{1, \nu}$. Hence there exists $b \in S_{a, v}-P$, i.e., there exists $b \in O^{*}-O, b \preccurlyeq a$ and $\alpha$ such that $Q(\alpha, b) \wedge \alpha_{1}=\nu$.

We now present three theorems relating the complexity of the functions $\left\{\alpha_{y}\right\}_{y \prec a}$ in a hierarchy to the ordering of $\{y: y \preccurlyeq a\}$. The third, and most important depends essentially on Theorem 1.8 .

THEOREM 2.2. There exists a partial recursive function $f$ such that if $a \in O^{*}$ and $H(\alpha, a), b_{1} \preccurlyeq b_{2} \preccurlyeq a$, then $f\left(b_{1}, b_{2}\right)$ is a Gödel number of $\alpha_{b_{1}}$ to be recursive in $\alpha_{b_{2}}$.

Proof. $b_{1} \preccurlyeq b_{2}$ implies that there exist sequence numbers $s$ and $m$ with the following properties:

(a) $b_{1}=(s)_{0} \prec(s)_{1} \prec(s)_{2} \cdots \prec(s)_{\operatorname{Lh}(s)-1}=b_{2}$;

(b) For each $i<\operatorname{Lh}(s)-1,(s)_{i+1}=2^{(s)_{i}}$ or $(s)_{i+1}=3 \cdot 5^{\left((s)_{i+1}\right)_{2}}$ and $\left\{(s)_{i+1,2}\right\}\left(m_{i+1}\right)$ $=(s)_{i}$.

We can express these properties of $b_{1}, b_{2}, s, m$ by $S\left(b_{1}, b_{2}, s, m\right)$ where $S$ is recursively enumerable. Hence there exist $g, h$ partial recursive such that $b_{1} \preccurlyeq b_{2}$ $\rightarrow S\left(b_{1}, b_{2}, g\left(b_{1}, b_{2}\right), h\left(b_{1}, b_{2}\right)\right)$. This last remark follows by the usual uniformization argument for recursively enumerable sets. Now suppose $b_{1} \preccurlyeq b_{2}, s=g\left(b_{1}, b_{2}\right)$, $m=h\left(b_{1}, b_{2}\right)$. Let $\alpha_{(s)_{t}}=\alpha_{i}$. It is sufficient to show how to find recursively a Gödel number of $\alpha_{i}$ in $\alpha_{i+1}$. If $(s)_{i+1}=2^{(s)_{i}}$ then $\alpha_{i}=\{k\}^{\alpha_{i+1}}$ where $k$ is a uniform Gödel number of $A$ in $A^{\prime}$. If $(s)_{i+1}=\left\{(s)_{i+1,2}\right\}\left((m)_{i+1}\right)$, then $\alpha_{i}(n)=\alpha_{i+1}\left(2^{m_{i+1}} \cdot 3^{n}\right)$ so $\alpha_{i}=\left\{j\left(m_{i+1}\right)\right\}^{\alpha_{i+1}}$ where $j$ is a recursive function such that $j(m)$ is a uniform Gödel number of $\left\{n: 2^{m} \cdot 3^{n} \in A\right\}$ in $A$. In either case we can recursively determine a Gödel number of $\alpha_{i}$ in $\alpha_{i+1}$. This proves the theorem.

In the following we write ' $\leqq_{R}$ ' for 'is recursive in', ' $=_{k}$ ' for 'has the same recursive degree as', ' $<_{R}$ ' for 'has lower recursive degree than'. Similarly, we use ' $\leqq_{H}$ ' for 'hyperarithmetic in', ' $=_{H}$ ' for 'has the same hyperdegree as', and ' $<_{H}$ ' for 'has lower hyperdegree than'.

CoROllary 2.3. If $a \in O^{*}$ and $H(\alpha, a)$, then $\alpha={ }_{R} \alpha_{a}$.

Proof. Obviously $\alpha_{a} \leqq_{R} \alpha$. Let $f$ be partial recursive and satisfy the conclusion of Theorem 2.2. Then $\alpha(y, z)=1$ if $y \npreceq a . \alpha(y, z)=\{f(y, a)\}^{\alpha}{ }^{\alpha}(z)$ if $y \preccurlyeq a$. Hence $\alpha$ is 
recursive in $\{y: y \preccurlyeq a\} \cup \alpha_{a}={ }_{R} \alpha_{a}$ since every recursively enumerable set is recursive in $\alpha_{a}$ if $2 \preccurlyeq a$, while if $a=1,\{y: y \preccurlyeq a\}$ is recursive.

THEOREM 2.4. If $a \in O^{*}, H(\alpha, a), p, q \preccurlyeq a,\{y: p \preccurlyeq y \preccurlyeq q\}$ has order type $\tau<\omega_{1}$ say $\tau=|n|_{\alpha_{p}}$ where $n \in O^{\alpha_{p}}$. Then $H_{n}^{\alpha}={ }_{R} \alpha_{q}$.

Proof. This is proved by induction on $\tau$ as in the uniqueness proof of Spector [17]. We omit the details.

THEOREM 2.5. Suppose $a \in O^{*}-O, H(\alpha, a), p, q \preccurlyeq a$, and $\{y: p \preccurlyeq y \preccurlyeq q\}$ is not well ordered. Then everything hyperarithmetic in $\alpha_{p}$ is recursive in $\alpha_{q}$.

Proof. We use a lemma due to Enderton and Putnam. We outline the proof, as it has not appeared.

Lemma Suppose $3 \cdot 5^{e} \in O,(\forall n)\left(H_{\{e)(n)}\right.$ is recursive in $\left.S\right)$. Then $H_{3.5^{e}}$ is recursive in $S^{\prime \prime \prime}$.

Proof. Let $\alpha^{n}$ be the unique hierarchy for $\{e\}(n)$ with $\alpha_{1}^{n} \equiv 1$. Note that $\alpha_{\{e\}(n)}^{n}$ is the characteristic function of $H_{\{e\}(n)}$ and that $\alpha^{n}={ }_{R} \alpha_{\{e\}(n)}^{n}$ by Corollary 2.3. Hence, for each $n, H_{\{e\}(n)} \leqq_{R} S$ iff $\alpha^{n} \leqq_{R} S$.

If, for all $n, H_{\{e\}(n)} \leqq_{R} S$, then

$$
\begin{aligned}
& n \in H_{3.5^{e}} \leftrightarrow(n)_{1} \in H_{\{e\}\left((n)_{0}\right)} \\
& \leftrightarrow(\forall \alpha)\left(H\left(\alpha,\{e\}\left((n)_{0}\right) \wedge \alpha_{1}=1 \rightarrow \alpha\left(\{e\}(n)_{0}\right),(n)_{1}\right)=0\right) \\
& \leftrightarrow(\exists \alpha)\left(H\left(\alpha,\{e\}\left(\left(n_{0}\right) \wedge \alpha_{1}=1 \wedge \alpha\left(\{e\}(n)_{0}\right),(n)_{1}\right)=0\right)\right. \\
& \leftrightarrow(\forall \alpha)\left(\alpha \leqq_{R} S \wedge H\left(\alpha,\{e\}\left((n)_{0}\right)\right) \wedge \alpha_{1}=1 \rightarrow \alpha\left(\{e\}\left((n)_{0}\right),(n)_{1}\right)=0\right) \\
& \leftrightarrow(\exists \alpha)\left(\alpha \varliminf_{R} S \wedge H\left(\alpha,\{e\}\left((n)_{0}\right) \wedge \alpha_{1}=1 \wedge \alpha\left(\{e\}(n)_{0}\right),(n)_{1}\right)=0\right) .
\end{aligned}
$$

By writing $(\forall \alpha)\left(\alpha \leqq_{R} S \wedge H\left(\alpha,\{e\}\left((n)_{0}\right)\right)\right.$ etc.) as $(\forall e)\left(\{e\}^{S}\right.$ is total etc. $)$, one obtains one four-quantifier form of $H_{3.5}$ e relative to $S$. The other is obtained similarly from the expression $(\exists \alpha)\left(\alpha \varliminf_{R} S \wedge H\left(\alpha,\{e\}\left((n)_{0}\right)\right.\right.$, etc.). Hence $H_{3.5^{e}} \leqq_{R} S^{m}$ by Post's Theorem.

Proof of theorem. Let $\alpha_{p}=\nu$. Suppose $n \in O^{\nu}$. We prove by induction on $|n|$ that $H_{n}^{v}$ is recursive in $\alpha_{c}$ for all $c$ such that $p \preccurlyeq c \preccurlyeq q$ and $\{y: p \preccurlyeq y \preccurlyeq q\}$ is not well ordered. For $n=1$ this is true by Theorem 2.2 since $H_{1}^{\nu}=\nu$. Suppose it is true for $n$. We will show that it is true for $m=2^{n}$. Given $c$ such that $p \preccurlyeq c \preccurlyeq q$ and $\{y: p \preccurlyeq y \preccurlyeq c\}$ is not well ordered, we must show that $H_{m}^{v}$ is recursive in $\alpha_{c}$.

If $\{y: p \preccurlyeq y \preccurlyeq c\}$ is not well ordered, then it has order type $\omega_{1} \cdot(1+\eta)+\alpha^{\prime}$ where $\alpha^{\prime}<\omega_{1}$. Choose $d \prec c$ such that $\{y: p \preccurlyeq y \preccurlyeq d\}$ and $\{y: d \preccurlyeq y \preccurlyeq c\}$ are not well ordered. Then by the hypothesis for $n, H_{n}^{\nu}$ is recursive in $\alpha_{d}$. Hence $H_{m}^{\nu}=\left(H_{n}^{\nu}\right)^{\prime}$ is recursive in $\left(\alpha_{d}\right)^{\prime}=\alpha_{2 d}$. Since $2^{d} \prec c, \alpha_{2 d}$ is recursive in $\alpha_{c}$. Using the transitivity of recursiveness we obtain that $H_{m}^{\nu}$ is recursive in $\alpha_{c}$.

Now suppose $n=3 \cdot 5^{e} \in O^{\nu}$. Let $f(i)=\{e\}^{v}(i)$ and suppose that for each $i, H_{f(i)}^{v}$ is recursive in $\alpha_{c}$ for each $c$ such that $p \preccurlyeq c \preccurlyeq q$ and $\{y: p \preccurlyeq y \preccurlyeq c\}$ is not well ordered. 
Given $c$ such that $p \preccurlyeq c \preccurlyeq q$ and $\{y: p \preccurlyeq y \preccurlyeq c\}$ is not well ordered, we must show that $H_{3.5}^{v}$ is recursive in $\alpha_{c}$.

Pick $d$ such that $d \prec c$ and $\{y: p \preccurlyeq y \preccurlyeq d\}$ is not well ordered and $\{y: d \preccurlyeq y \preccurlyeq c\}$ is not well ordered. Then for each $i, H_{f(i)}^{v}$ is recursive in $\alpha_{d}$; so by the relativization of Enderton's lemma to $\nu, H_{3.5}^{v}$ is recursive in $\left(\alpha_{d}\right)^{\prime \prime \prime}=\alpha_{g}$ where $d \prec g \prec c,|g|=|d+4|$. Since $g \prec c$, it follows that $H_{3.5}^{v}$ is recursive in $\alpha_{c}$.

By induction on $|n|$ it follows that for any $c, p \preccurlyeq c \preccurlyeq q$ with $\{y: p \preccurlyeq y \preccurlyeq q\}$ not well ordered, $H_{n}^{v}$ is recursive in $\alpha_{c}$ for all $n \in O^{v}$. Hence everything hyperarithmetic in $\alpha_{p}$ is recursive in $\alpha_{c}$, which proves the theorem.

Note that Theorem 2.5 is also a consequence of Theorems 2.2 and 2.4 if $\omega_{1}^{\nu}=\omega_{1}$. For the case where $\omega_{1}^{\nu}>\omega_{1}$ we need the proof given.

A particular consequence of Theorem 2.5 is that if $a \in O^{*}-O$ and $H(\alpha, a)$ $\wedge \alpha_{1}=1$, then every hyperarithmetic set is recursive in $\alpha$, and so $\alpha$ is not hyperarithmetic. Hence the statement ' $\left.(\forall a)(a \in O) \rightarrow(\exists \alpha)\left(H(\alpha, a) \wedge \alpha_{1}=1\right)\right)$ ' cannot be proved solely by the $\Sigma_{1}^{1}$ axiom of dependent choices, for it does not relativize to $O^{*}$. This explains the earlier remark about the difference between this section and section one.

We now consider a completeness property of functions in a hierarchy. Suppose $a \in O^{*}-O$ and $H(\alpha, a)$. Say that $\alpha$ has a gap if there exists a function $\beta$ with the following properties: (a) for all $y \preccurlyeq a, \alpha_{y} \leqq_{H} \beta$ or $\beta \leqq_{H} \alpha_{y}$; (b) for all $y \preccurlyeq a, \beta \neq_{H} \alpha_{y}$; (c) $\beta \varliminf_{H} \alpha_{1},\left.\alpha_{a}\right|_{H} \beta$.

Note that if $\beta$ has these properties with respect to $\alpha$, then either (1)

$$
\left\{y: y \preccurlyeq a \wedge \beta \leqq_{H} \alpha_{y}\right\}=A
$$

is nonempty and has no least element, or (2) $A$ is nonempty and has a least element $3 \cdot 5^{e}$ which is the first element of its equivalence class. If (2) is the case we have that for each $n, \alpha_{\{e\}(n)} \leqq_{H} \beta, \beta \leqq_{H} \alpha_{3.5^{e}}$, but $\alpha_{3.5^{e}} \varliminf_{H} \beta$.

Now we show

THEOREM 2.6. If $a \in O^{*}-O, Q(\alpha, a)$ and $(\forall y)\left(y \preccurlyeq a \rightarrow \beta \leqq_{H} \alpha_{y}\right.$ or $\left.\alpha_{y} \leqq_{H} \beta\right)$, then either $\alpha_{1} \geqq_{H} \beta$, or $\alpha_{a} \leqq_{H} \beta$, or $\beta=_{H} \alpha_{y}$ for some $y \preccurlyeq a$. Hence $\alpha$ has no gaps.

Proof. It is sufficient to show that $(1) \rightarrow \neg Q(\alpha, a)$, and (2) $\rightarrow \neg Q(\alpha, a)$.

Suppose (1) holds. We claim $A=\left\{y: y \preccurlyeq a \wedge \beta \leqq_{R} \alpha_{y}\right\}$. Clearly

$$
A \supseteq\left\{y: y \preccurlyeq a \wedge \beta \leqq_{R} \alpha_{y}\right\} .
$$

Suppose $y \in A$. Since $A$ has no least element, by Theorem 1.8 we can choose $y^{\prime} \prec y, y^{\prime} \in A$, so that $\left\{u: y^{\prime} \preccurlyeq u \preccurlyeq y\right\}$ is not well ordered. Since $y^{\prime} \in A$, it follows that $\beta \leqq_{H} \alpha_{y^{\prime}}$. By Theorem 2.5 and the choice of $y^{\prime}$, everything is hyperarithmetic in $\alpha_{y^{\prime}}$ and hence $\beta$ is recursive in $\alpha_{y}$. This shows $A \subseteq\left\{y: y \preccurlyeq a \wedge \beta \leqq_{R} \alpha_{y}\right\}$ and so

$$
A=\left\{y: y \preccurlyeq a \wedge \beta \leqq_{R} \alpha_{y}\right\}=\left\{y: y \preccurlyeq a \wedge\{e\}^{\alpha} \leqq_{R} \alpha_{y}\right\},
$$

where $e$ is some Gödel number of $\beta$ in $\alpha$. This shows that $A$ is arithmetic, a fortiori 
hyperarithmetic in $\alpha$. Since $A$ has no least element and $A \subseteq\{y: y \preccurlyeq a\}$ we have $\neg Q(\alpha, a)$.

Suppose (2) holds. Note that if $H(\alpha, a), b \preccurlyeq a$ and we let $\alpha^{b}(x, y)=\alpha(x, y)$ for $x \preccurlyeq b, \alpha^{b}(x, y)=1$ for $x \preccurlyeq b$, then $H\left(\alpha^{b}, b\right)$ and $\alpha^{b} \leqq_{H} \alpha$. If $\alpha^{b}$ is the only hierarchy for $b$ with $\alpha_{1}^{b}=\alpha_{1}$ and $\alpha^{b} \leqq_{H} \alpha$ we would have

$$
\begin{aligned}
\left(\alpha_{3.5^{e}}(n)=0 \leftrightarrow\right. & (\exists \nu)\left(\nu \leqq_{H} \beta \wedge H\left(\nu,\{e\}\left((n)_{0}\right) \wedge \nu_{1}=\alpha_{1} \wedge \nu\left(\{e\}\left((n)_{0}\right),(n)_{1}\right)=0\right)\right) \\
& \left.\leftrightarrow\left((\forall \nu)\left(\nu \leqq_{H} \beta \wedge H\left(\nu,\{e\}(n)_{0}\right)\right) \wedge \nu_{1}=\alpha_{1} \rightarrow \nu\left(\{e\}\left((n)_{0}\right),(n)_{1}\right)=0\right)\right)
\end{aligned}
$$

Since $\alpha_{1} \leqq_{H} \alpha_{\{e\}(1)} \leqq_{H} \beta$, the above equivalences show that $\alpha_{3.5^{e}} \in \Pi_{1}^{1(\beta)}$ and that $\alpha_{3.5^{e}} \in \Sigma_{1}^{1(\beta)}$, respectively, i.e. $\alpha_{3.5^{e}} \leqq_{H} \beta$ which contradicts (2). Hence there exist $\nu, \delta$ such that $\nu \leqq_{H} \alpha, \delta \leqq_{H} \alpha$ for some $b \preccurlyeq a, H(\nu, b), H(\delta, b)$, and $\nu_{1}=\delta_{1}=\alpha_{1}$, but $\nu \neq \delta$. Let $D_{v, \delta}=\left\{y: y \preccurlyeq b \wedge \nu_{y} \neq \delta_{y}\right\}$. $D_{v, \delta}$ has no least element since $\nu$ and $\delta$ are hierarchies with the same initial function, and the least element of $D_{v, \delta}$ could not be a successor or a limit. $D_{v, \delta} \subset\{y: y \preccurlyeq a\}$ and $D_{v, \delta} \leqq_{H} \alpha$. This shows $\neg Q(a, \alpha)$. So $(2) \rightarrow \neg Q(\alpha, a)$, and the proof of the theorem is complete.

Corollary 2.7. If $Q(\alpha, a), Q(\beta, a), \alpha_{1}=\beta_{1}, b \preccurlyeq a$, and $\alpha_{b} \neq \beta_{b}$, then $\alpha_{b}{ }_{H} \beta$, $\beta_{b} \leqq{ }_{H} \alpha$.

Proof. We have seen that $\alpha^{b}$ (respectively $\beta^{b}$ ) is the only hierarchy for $b$ which is hyperarithmetic in $\alpha$ (respectively $\beta$ ). Since $\alpha_{b}={ }_{R} \alpha^{b}, \beta_{b}={ }_{R} \beta^{b}$, and $\alpha_{b} \neq \beta_{b} \rightarrow \alpha^{b} \neq \beta^{b}$, it follows that $\beta_{b} \hbar_{H} \alpha, \alpha_{b} \aleph_{H} \beta$. We will use this corollary in the proof of Theorem 2.11 .

CoROllaRy 2.8. If $Q(\alpha, a)$ then (i) if $b \prec a, b \notin E(a)$ then $\alpha_{b}$ is a greatest lower bound in hyperdegree for the hyperdegrees of the functions of the set $\left\{\alpha_{y}: b \preccurlyeq y \preccurlyeq a\right.$ and $\{u: b \preccurlyeq u \preccurlyeq y\}$ is not well ordered $\}$; (ii) if $3 \cdot 5^{e} \preccurlyeq a$ and $3 \cdot 5^{e}$ is the first element of its equivalence class, then $\alpha_{3.5^{e}}$ is a minimal upper bound in hyperdegree for the hyperdegrees of the functions in the set $\left\{\alpha_{(e)(n)}: n \in \omega\right\}$; (iii) suppose that $A \subseteq\{y: y \preccurlyeq a\}$ has no least element, that $y \in A \wedge y \prec z \preccurlyeq a \rightarrow z \in A$ and that $B=\{y: y \preccurlyeq a\}-A$ has no greatest equivalence class (one might say in this case that the sets $A$ and $B$ define an irrational cut in $\{y: y \preccurlyeq a\})$, then the hyperdegrees of the functions in the set $\left\{\alpha_{y}: y \in A\right\}$ have no greatest lower bound, and the hyperdegrees of the functions in the set $\left\{\alpha_{y}: y \in B\right\}$ have no least upper bound.

Proof. (i) Suppose that if $b \preccurlyeq y$ and $\{u: b \preccurlyeq u \preccurlyeq y\}$ is not well ordered then $\beta \leqq_{H} \alpha_{y}$. The first assertion will follow if we can show that $\beta \leqq_{H} \alpha_{b}$. If $B=\left\{y: y \preccurlyeq a \wedge \beta \leqq_{H} \alpha_{y}\right\}$, then $B \supseteq\{y: b \preccurlyeq y \preccurlyeq a \wedge\{u: b \preccurlyeq u \preccurlyeq y\}$ is not well ordered $\}$. If equality holds then $B$ has no least element and by the argument of the theorem, $B \leqq_{H} \alpha$. Hence $\neg Q(\alpha, a)$. Therefore the inclusion is strict and so there must exist $c \preccurlyeq a$ such that $\beta \leqq_{H} \alpha_{c}$ and either $c \preccurlyeq b$ or $\{u: b \preccurlyeq u \preccurlyeq c\}$ is well ordered. In either case it follows that $\beta \leqq_{H} \alpha_{b}$. (ii) This result is the contrapositive of the implication ' $(2) \rightarrow \neg Q(\alpha, a)$ '. (iii) If $\nu$ is a greatest lower bound in hyperdegree for the hyperdegrees of the functions of the set $\left\{\alpha_{y}: y \in A\right\}$ or a least upper bound in hyperdegree for the 
hyperdegrees of the functions of the set $\left\{\alpha_{y}: y \notin A\right\}$, then $A=\left\{y: y \preccurlyeq a \wedge \beta \leqq_{H} \alpha_{y}\right\}$ which the proof of the theorem shows is impossible.

The following extracts a hierarchy free statement from this result. We here abbreviate 'hyperdegree' by ' $h-d e g$ '.

COROLlaRy 2.9. Suppose $\omega_{1}^{\alpha}=\omega_{1}$ and all hyperarithmetic sets are recursive in $\alpha$. Then there exists a densely ordered set of h-degs $C$ all less than the $h$-deg of $\alpha$ and such that the h-deg of the hyperarithmetic sets is in $C$, and any h-deg less than some $h$-deg in $C$ and comparable to each member of $C$ is equal to some member of $C$.

Proof. Let $S_{a}=\left\{b: b \preccurlyeq a \wedge(\exists \beta)\left(Q(\beta, b) \wedge \beta_{1}=1 \wedge \beta\right.\right.$ is recursive in $\left.\left.\alpha\right)\right\}$. Since all hyperarithmetic sets are recursive in $\alpha$, it follows that $S_{a} \supset\{b: b \preccurlyeq a\} \cap O$. By the argument of Theorem 2.1, there exists $b \in O^{*}-O \cap S_{a}$. The corollary follows immediately from this and Corollary 2.8 .

COROLlary 2.10. Suppose $\alpha$ has minimal hyperdegree. Then if $y \in O$ not all hyperarithmetic sets are recursive in $H_{y}^{\alpha}$.

Proof. If $\alpha$ has minimal hyperdegree then certainly $\omega_{1}^{\alpha}=\omega_{1}$. Hence Corollary 2.10 follows from Corollary 2.9 applied to $H_{y}^{\alpha}$.

The hypothesis in the preceding is known to be nonvacuous according to a result of Gandy and Sacks (informally circulated notes).

So far we have concentrated attention on properties of individual solutions of $Q(\alpha, a)$ for $a \in O^{*}-O$. We now study pairs of solutions $\alpha, \beta$ of such. Let $(\alpha \cup \beta)(x)$ $=2^{\alpha(x)} \cdot 3^{\beta(x)}$.

THEOREM 2.11. (i) Suppose $a \in O^{*}-O$ and $(\exists \alpha)\left(Q(\alpha, a) \wedge \alpha_{1}=1\right)$. Then

$$
(\exists \alpha)(\exists \beta)\left(Q(\alpha, a) \wedge Q(\beta, a) \wedge \alpha_{1} \equiv \beta_{1} \equiv 1 \wedge \alpha \neq \beta \wedge \omega_{1}^{\alpha \cup \beta}=\omega_{1}\right) .
$$

(ii) Suppose $Q(\alpha, a) \wedge Q(\beta, a) \wedge \alpha_{1}=\beta_{1}=1, \alpha \neq \beta \wedge \omega_{1}^{\alpha \cup \beta}=\omega_{1}$. Let

$$
D_{\alpha, \beta}=\left\{y: y \preccurlyeq a \wedge \alpha_{y} \neq \beta_{y}\right\} \text {. }
$$

Then for no function $\nu$ do we have (1) $y \preccurlyeq a \wedge y \notin D_{\alpha, \beta} \rightarrow \alpha_{y} \leqq_{H} \nu$, and (2) $\nu \leqq_{H} \alpha$, and (3) $\nu \varliminf_{H} \beta$. Hence $\alpha$ and $\beta$ have no greatest lower bound in hyperdegree.

Proof. (i) If $(\exists ! \alpha)\left(Q(\alpha, a) \wedge \alpha_{1}=1\right)$ then this $\alpha$ is hyperarithmetic. So if $a \in O^{*}-O$ and $(\exists \alpha)\left(Q(\alpha, a) \wedge \alpha_{1}=1\right)$ then $(\exists \alpha)(\exists \beta)\left(Q(\alpha, a) \wedge Q(\beta, a) \wedge \alpha_{1}=\beta_{1}=1 \wedge \alpha \neq \beta\right)$. By Gandy's Basis Result [6] we can choose $\alpha$ and $\beta$ so that $\alpha \cup \beta<{ }_{H} O$ and hence $\omega_{1}^{\alpha \cup \beta}=\omega_{1}$. (ii) Suppose $\nu$ has the property that $\nu \leqq_{H} \alpha$, and if $y \preccurlyeq a$ and $y \notin D_{\alpha, \beta}$, then $\alpha_{y} \leqq_{H} \nu$. We will show that for some $z \in D_{\alpha, \beta}, \alpha_{z} \leqq_{H} \nu$. This shows $\nu \varliminf_{H} \beta$ since, by Corollary 2.7, $\alpha_{z}{ }_{H} \beta$ if $z \in D_{\alpha, \beta}$.

To prove that such a $z$ exists it is sufficient to show that there exists $\nu^{\prime} \leqq_{H} \nu$ such that if $y \preccurlyeq a$ and $y \notin D_{\alpha, \beta}$ then $\alpha_{y} \leqq_{R} \nu^{\prime}$. For if $\nu^{\prime}$ has this property and for no $z \in D_{\alpha, \beta}$ is $\alpha_{z} \leqq_{R} \nu^{\prime} \leqq_{H} \nu$, then $D_{\alpha, \beta}=\left\{y: y \preccurlyeq a \wedge \alpha_{y} \leqq_{R} \nu^{\prime}\right\}$ and hence $D_{\alpha, \beta} \leqq_{H} \alpha$, and $\neg Q(\alpha, a)$, since $D_{\alpha, \beta}$ has no least element. Hence if such a $\nu^{\prime}$ exists, then there exists a $z$ with the desired property. 
To prove the existence of $\nu^{\prime}$ we proceed as follows. Since $(\forall y)\left(y \preccurlyeq a \wedge y \notin D_{\alpha, \beta}\right.$ $\left.\rightarrow \alpha_{y} \leqq_{H} \nu\right)$,

$$
(\forall y)\left(y \preccurlyeq a \wedge y \notin D_{\alpha, \beta} \rightarrow(\exists e)(\exists u)\left(u \in O^{v} \wedge\{e\}^{\delta}=\alpha_{y}\right)\right), \quad \delta=H_{u}^{v} .
$$

$D_{\alpha, \beta} \leqq_{H} \alpha \cup \beta$, and $\nu \leqq_{H} \alpha \cup \beta$; so we can write (1) in the form

$$
(\forall y)(\exists e)(\exists u) P(y, e, u, \alpha \cup \beta),
$$

where the expression $P(y, e, u, \alpha \cup \beta)$ is $\Pi_{1}^{1, \alpha \cup \beta}$. Hence

$$
\begin{gathered}
(\exists \zeta)\left(\zeta \leqq_{H} \alpha \cup \beta \wedge(\forall y) P\left(y, \zeta(y)_{0}, \zeta(y)_{1}, \alpha \cup \beta\right)\right), \\
(\exists \zeta)\left(\zeta \leqq_{H} \alpha \cup \beta \wedge(\forall y)\left(y \preccurlyeq a \wedge y \notin D_{\alpha, \beta} \rightarrow \zeta(y)_{0} \in O^{v} \wedge\left\{\zeta(y)_{1}\right\}^{\delta}=\alpha_{y}\right)\right), \\
\delta=H_{\zeta(y)_{0}}^{v}
\end{gathered}
$$

We claim that for some $u^{\prime} \in O^{v}$, if $y \preccurlyeq a$ and $y \notin D_{\alpha, \beta}$ then $\left|\zeta(y)_{0}\right| \leqq\left|u^{\prime}\right|$. Otherwise $O^{v} \leqq_{H} \alpha \cup \beta$ which contradicts the fact that $\omega_{1}^{\alpha \cup \beta}=\omega_{1}$. For $O^{v}$ is trivially $\Pi_{1}^{1, \alpha \cup \beta}$ since $\nu \leqq_{H} \alpha \cup \beta$. If the range of $\zeta$ is unbounded in $O^{v}$ then we would also have $O^{v} \in \Sigma_{1}^{1, \alpha \cup \beta}$, for then

(5) $n \in O^{v} \leftrightarrow\left(\left\{z: z \preccurlyeq o^{v} n\right\}\right.$ is linearly ordered by $\preccurlyeq o^{\nu} \wedge(\exists y)(\exists f)(y \preccurlyeq a$ $\wedge y \notin D_{\alpha, \beta} \wedge f$ is an isomorphism of $\left\{z: z \preccurlyeq o^{v} n\right\}$ into $\left.\left.\left\{z: z \preccurlyeq o^{v} \zeta(y)_{0}\right\}\right)\right)$.

So for some $u^{\prime} \in O^{\nu}$, if $y \preccurlyeq a$ and $y \notin D_{\alpha, \beta}$ then $\left|\zeta(y)_{0}\right| \leqq\left|u^{\prime}\right|$. Hence by the property (4) of $\zeta$, if $y \preccurlyeq a$ and $y \notin D_{\alpha, \beta}, \alpha_{y} \leqq_{R} H_{u^{\prime}}^{v}$ so we can take $\nu^{\prime}=H_{u^{\prime}}^{v}$. This completes the proof.

Our next theorem is related to the classical result that any uncountable analytic set of real numbers contains a perfect subset. For a given $\Sigma_{1}^{1}$ set $S$ of numbertheoretic functions containing a nonhyperarithmetic function, we obtain the existence of a subset $T$ of $S$ which has the cardinality of the continuum and such that any two distinct members of $T$ are hyperarithmetically incomparable. Let $\alpha \cap_{H} \beta=\left\{\nu: \nu \varliminf_{H} \alpha \wedge \nu \leqq_{H} \beta\right\}$. Let $\alpha \cap_{R} \beta=\left\{\nu: \nu \varliminf_{R} \alpha \wedge \nu \varliminf_{R} \beta\right\}$.

THEOREM 2.12. Let $R$ be recursive. Suppose $(\exists \beta)(\exists \nu)(\forall x)(R(\bar{\beta}(x), \bar{\nu}(x)) \wedge \beta \notin H A)$. Then there exists $D \subseteq\{\beta:(\exists \nu)(\forall x) R(\bar{\beta}(x), \bar{\nu}(x)) \wedge \beta \notin H A\}$, such that $\overline{\bar{D}}=2^{\mathrm{x}_{0}}$, and $\alpha, \beta \in D, \alpha \neq \beta \rightarrow \alpha \cap_{H} \beta \subseteq H A$. If $\alpha \notin H A$, there exists $\beta \in D$, such that $\beta<_{H} O^{\alpha}$ $\wedge \alpha \cap_{H} \beta \subseteq H A$.

Proof. First we prove a slightly weaker result. Then we strengthen it to the conclusion of Theorem 2.12. The proof makes no use of hierarchies.

THEOREM $2.12^{\prime}$. Let $R$ be recursive. Suppose $(\exists \beta)(\forall x) R(\bar{\beta}(x)), \neg(\exists \beta)_{H A}(\forall x) R(\bar{\beta}(x))$. Then there exists a set $C \subseteq\{\beta:(\forall x) R(\bar{\beta}(x))\}$ such that $\overline{\bar{C}}=2{ }^{\kappa_{0}}$, and $\alpha, \beta \in C$, $\alpha \not \equiv \beta \rightarrow \alpha \cap_{R} \beta \subseteq H A$.

Proof. Let $T_{R}=\{s:(\exists \beta)(\beta \supset s \wedge(\forall x) R(\widetilde{\beta}(x)))\}$. Note that for any function $\beta$, $(\forall x) R(\bar{\beta}(x)) \leftrightarrow(\forall x)\left(\bar{\beta}(x) \in T_{R}\right)$. We will construct a subset $T_{R}^{\prime}$ of $T_{R}$ such that $C=\left\{\beta:(\forall x)\left(\beta(x) \in T_{R}^{\prime}\right)\right\}$ satisfies the conclusion of Theorem 2.12'. We will define 
$T_{R^{\prime}}=\left\{s:(\exists n)(\exists t)\left(t \in T_{n} \wedge s \subset t\right)\right\}$. The sets $T_{n}$ will have the following properties: (i) $T_{0}=\{1\}$. (ii) $\overline{\bar{T}}_{n}=2^{n} ; t \in T_{n}$ implies there exist $t_{1}, t_{2} \in T_{n+1}, t_{1} \neq t_{2}, t_{1} \supset t, t_{2} \supset t$; $T_{n} \subset T_{R}$. (iii) If $s, t \in T_{n}, s \neq t$, and $n \geqq m$ then

$$
\begin{aligned}
(\forall \alpha)(\forall \beta)(\alpha \supset s \wedge \beta \supset t \wedge(\forall x) R(\bar{\beta}(x)) & \\
\wedge & (\forall x) R(\bar{\alpha}(x)) \wedge\left\{(m)_{0}\right\}^{\alpha} \equiv\left\{(m)_{1}\right\}^{\beta} \rightarrow\left\{(m)_{0}\right\}^{\alpha} \in H A .
\end{aligned}
$$

Suppose we have constructed the sets $T_{n}$ with these properties and defined $C$ as above. Clearly $\overline{\bar{C}}=2^{\aleph_{0}}$ by (ii), and $C \subseteq\{\beta:(\forall x) R(\bar{\beta}(x))\}$. Suppose $\alpha, \beta \in C$ and $\alpha \not \equiv \beta$. Let $m$ be an arbitrary integer. Let $n=\max (m+1, \mu k(\alpha(k) \neq \beta(k)))$. Then by (ii) and choice of $n$, there exist $s, t \in T_{n}$, such that $s \neq t$ and $\bar{\alpha}(l h(s))=s, \bar{\beta}(l h(t))=t$. Hence by (iii), $\left\{(m)_{0}\right\}^{\alpha} \equiv\left\{(m)_{1}\right\}^{\beta} \rightarrow\left\{(m)_{0}\right\}^{\alpha} \in H A$. Since $m$ was arbitrary we must have $\alpha \cap_{R} \beta \subseteq H A$.

The sets $T_{n}$ are defined inductively. For each $t \in T_{n}$ we choose $t_{1}, t_{2} \in T_{R}$ such that $t_{1} \supset t, t_{2} \supset t$, and $t_{1} \neq t_{2}$. This is always possible since $R$ has no hyperarithmetic solutions. Call the resulting set $T_{n}^{\prime}$. We now need the following lemma.

Lemma. Given a triple of integers $\langle s, t, m\rangle$ where $s, t \in T_{R}$ and $m$ is arbitrary, we can find a triple $\left\langle s^{\prime}, t^{\prime}, m\right\rangle$ such that $s \subset s^{\prime} \in T_{R}, t \subset t^{\prime} \in T_{R}$, and

$$
\begin{aligned}
(\forall \alpha)(\forall \beta)\left(\alpha \supset s^{\prime} \wedge \beta \supset t^{\prime} \wedge(\forall x)(R(\bar{\alpha}(x))\right. & \wedge R(\bar{\beta}(x))) \\
& \left.\wedge\left\{(m)_{0}\right\}^{\alpha} \equiv\left\{(m)_{1}\right\}^{\beta} \rightarrow\left\{(m)_{0}\right\}^{\alpha} \in H A\right) .
\end{aligned}
$$

Assume the lemma holds. Fix some list of the triples $\langle s, t, m\rangle$ with $s, t \in T_{n}^{\prime}$ and $m \leqq n+1$, and apply the lemma to the first triple in the list $\langle u, v, k\rangle$ to obtain a new triple $\left\langle u^{\prime}, v^{\prime}, k\right\rangle$ satisfying the underlined property with respect to $\langle u, v, k\rangle$. Then replace all occurrences of $u$ and $v$ in triples in the list by $u^{\prime}$ and $v^{\prime}$ respectively. Now apply the lemma to the second triple in the modified list and make the corresponding replacements. If we repeat this process until we have come to the end of the list, the set of sequences obtained will form a set $T_{n+1}$ with the required properties (ii) and (iii).

\section{Proof of Lemma.}

Case 1. $(\forall \alpha)(\forall \nu)\left(\alpha \supset s \wedge \nu \supset s \wedge(\forall x)(R(\bar{\alpha}(x)) \wedge R(\bar{\nu}(x)))\left\{(n)_{0}\right\}^{\alpha},\left\{(n)_{0}\right\}^{\nu}\right.$ are total $\left.\rightarrow\left\{(n)_{0}\right\}^{\alpha} \equiv\left\{(n)_{0}\right\}^{\gamma}\right)$. Then if $\alpha \supset s \wedge(\forall x) R(\bar{\alpha}(x)) \wedge\left\{(n)_{0}\right\}^{\alpha}$ is total, then $\left\{(n)_{0}\right\}^{\alpha}$ is hyperarithmetic since

$$
\left\{(n)_{0}\right\}^{\alpha}(x)=y \leftrightarrow(\forall v)\left(\nu \supset s \wedge(\forall x) R(\bar{v}(x)) \wedge\left\{(n)_{0}\right\}^{v} \text { is total } \rightarrow\left\{(n)_{0}\right\}^{\nu}(x)=y\right) .
$$

Let $s^{\prime}=\mu y\left(y \in T_{R} \wedge y \supset s\right), t^{\prime}=\mu z\left(z \in T_{R} \wedge z \supset t\right)$.

\section{Case 2.}

$$
(\exists u)(\exists \alpha)(\exists \beta)\left(\alpha \supset s \wedge \beta \supset s \wedge(\forall x)\left(R(\bar{\alpha}(x)) \wedge R(\bar{\beta}(x)) \wedge\left\{(n)_{0}\right\}^{\alpha}(u) \neq\left\{(n)_{0}\right\}^{\beta}(u)\right)\right) .
$$

Choose $u$ with this property. If for all $t^{\prime \prime} \supset t, t^{\prime \prime} \in T_{R},\{(n)\}^{t^{\prime \prime}}(u)$ is undefined, then define $s^{\prime}$ and $t^{\prime}$ as in Case 1. Otherwise choose $p, q \in T_{R}$ so that $s \subset p, s \subset q$, and $\left\{(n)_{0}\right\}^{p}(u) \neq\left\{(n)_{0}\right\}^{q}(u)$. Let $t^{\prime}=\mu y\left(y \supset t \wedge y \in T_{R} \wedge\left\{(n)_{1}\right\}^{y}(u)\right.$ is defined). Let $s^{\prime}=p$ if 
$\left\{(n)_{0}\right\}^{p}(u) \neq\left\{(n)_{1}\right\}^{t^{\prime}}(u)$; otherwise let $s^{\prime}=q$. In either Cases 1 or $2, s^{\prime}$ and $t^{\prime}$ satisfy the conclusion of the lemma.

This completes the proof of Theorem $2.12^{\prime}$.

We now complete the proof of Theorem 2.12. Write

$$
\alpha \notin H A \leftrightarrow(\exists v)(\forall x) N(\bar{\nu}(x), \bar{\alpha}(x)) ; \quad H(\alpha, a) \leftrightarrow(\exists v)(\forall x) H(\bar{\alpha}(x), \bar{\nu}(x), a)
$$

whence $H$ and $N$ are recursive.

Fix $a \in O^{*}-O$. Let

$$
\begin{aligned}
B=\{y: y \preccurlyeq a \wedge(\exists \alpha)(\exists \beta)(\exists v)(\exists \delta)(\forall x)(\forall u)(\forall v) H(\bar{\alpha}(x), \bar{\beta}(x), y) \\
\wedge N(\bar{\alpha}(1, u), \bar{\nu}(u)) \wedge R(\bar{\alpha}(1, v), \delta(v))\} .
\end{aligned}
$$

$B \in \Sigma_{1}^{1}$, and $B \supset O \cap\{y: y \preccurlyeq a\}$ which is a $\Pi_{1}^{1}$ path through $O$ and so a proper $\Pi_{1}^{1}$ set. Hence there exists $b \in B \cap O^{*}-O$.

Let

$$
F=\left\{f:(\forall x)\left(H\left(\bar{f}(x)_{0}, \bar{f}(x)_{1}, b\right) \wedge N\left(\bar{f}(1, x)_{0}, \bar{f}(x)_{2}\right) \wedge R\left(\bar{f}(1, x)_{0}, \bar{f}(x)_{3}\right)\right)\right\} .
$$

By Theorem $2.12^{\prime}$ we can choose $C \subset F, \overline{\bar{C}}=2^{x_{0}}$, with the property that $f, g \in C$, $f \neq g \rightarrow f \cap_{R} g \subseteq H A$. Let $D=\left\{h:(\exists f)\left(f \in C \wedge h=\lambda x f(1, x)_{0}\right)\right\}$. Clearly

$$
D \subseteq\{h:(\exists f)(\forall x) R(h(x), f(x)) \wedge h \notin H A\} .
$$

We will show that $\overline{\bar{D}}=2 \aleph^{\aleph_{0}}$, and that $h, j \in D, h \neq j \rightarrow h \cap_{H} j \subseteq H A$.

First note that if $f, g \in C, f \neq g$, then $\lambda x f(1, x)_{0} \neq \lambda x g(1, x)_{0}$. For if $\lambda x f(1, x)_{0}$ $=\lambda x g(1, x)_{0}$ then $\lambda x f(1, x)_{0} \leqq_{R} g$ and hence $\lambda x f(1, x)_{0} \in H A$ since $f, g \in C$ and $f \neq g$. But $f \in C$ implies $\lambda x f(1, x)_{0} \notin H A$, so we must have $\lambda x f(1, x)_{0} \neq \lambda x g(1, x)_{0}$. Thus $\overline{\bar{C}}=\overline{\bar{D}}=2^{\text {ro. }}$.

Suppose $h, j \in D, h \neq j$. Choose $f, g \in C$ such that $\lambda x f(1, x)_{0}=h, \lambda x g(1, x)_{0}=j$. Since $\lambda x f(x)_{0}$ and $\lambda x g(x)_{0}$ are hierarchies on $b \in O^{*}-O$ with initial functions $\lambda x f(1, x)_{0}$ and $\lambda x g(1, x)_{0}$ respectively, it follows that everything hyperarithmetic in $\lambda x f(1, x)_{0}$ is recursive in $f$, and everything hyperarithmetic in $\lambda x g(1, x)_{0}$ is recursive in $g$. Since $f, g \in C$ and $f \neq g$ (else $h=j$ ), it follows that $f \cap_{R} g \subseteq H A$. Hence $\lambda x f(1, x)_{0} \cap_{H} \lambda x g(1, x)_{0} \subseteq H A$, i.e., $h \cap_{H} j \subseteq H A$ as desired. Thus $D$ satisfies the first part of the conclusion of the theorem.

Proof of second part. Suppose $\nu \notin H A$. Let $T_{R^{\prime}}$ be defined as in the proof of Theorem 2.12'. Let $t_{0}=1$. Given $t_{n-1} \in T_{R^{\prime}}$, we let $t_{n}=\mu y\left(y \in T_{R^{\prime}} \wedge y \supset t_{n-1} \wedge(\exists u)\right.$ $\left.\left(\left\{(n)_{0}\right\}^{y}(u) \neq\left\{(n)_{1}\right\}^{v}(u)\right)\right)$ if such a $y$ exists, otherwise $t_{n}=\mu y\left(y \in T_{R^{\prime}} \wedge y \supset t_{n-1}\right)$. Under the first alternative, $\left\{(n)_{0}\right\}^{\beta} \neq\left\{(n)_{1}\right\}^{\nu}$ if $\beta \supset t_{n}$. Under the second, if $\beta \supset t_{n}$, and $\beta$ is a path through $T_{R^{\prime}}$ then, by the argument of Case 1 of the Lemma of Theorem $2.12^{\prime},\left\{(n)_{0}\right\}^{\beta}$ is hyperarithmetic if it is total, or $\left\{(n)_{1}\right\}^{v}$ is not total.

Define $\delta$ by the conditions $\delta\left(\operatorname{lh}\left(t_{n}\right)\right)=t_{n}$ for each $n$. $\delta$ is arithmetic in $T_{R^{\prime}} \cup \nu$, and $\delta$ is a path through $T_{R^{\prime}}$. Moreover, for each $n,\left\{(n)_{0}\right\}^{\delta}=\left\{(n)_{1}\right\}^{\nu} \rightarrow\left\{(n)_{0}\right\}^{\delta} \in H A$. Hence $\delta \cap_{R} \nu \subseteq H A$.

Let $\nu=O^{\alpha}$, and apply this construction to the set $F$ defined in the proof of the 
first part of the theorem. One obtains $f \in C \subseteq F$ such that $f \cap_{R} O^{\alpha} \subseteq H A$ and $f \leqq_{H} T_{R} \cup O^{\alpha}$. We will show that $\lambda x f(1, x)_{0}$ satisfies the second part of the conclusion of Theorem 2.12.

Since everything hyperarithmetic in $\lambda x f(1, x)_{0}$ (resp. $\alpha$ ) is recursive in $f$ (resp. $O^{\alpha}$ ), and $f \cap_{R} O^{\alpha} \subseteq H A$, it follows that $\lambda x f(1, x)_{0} \cap_{H} \alpha \subseteq H A$. Since $f \in C$, it follows that $\lambda x f(1, x)_{0} \in D$. It is not hard to show $T_{R^{\prime}}$ can be defined recursively in $O$. Using this fact we obtain $\lambda x f(1, x)_{0}<_{H} f \leqq_{H} T_{R^{\prime}} \cup O^{\alpha}=_{H} O \cup O^{\alpha}=_{H} O^{\alpha}$. This completes the proof $\left({ }^{3}\right)$.

COROLLARY 2.13. If $[\alpha]$ is any hyperdegree which contains some function $\alpha$ in which all hyperarithmetic sets are recursive, and, in addition, $0<[\alpha]<0^{\prime}$, then we can find a hyperdegree $[\beta]$ with $0<[\beta]<0^{\prime}$ and $[\alpha] \cap[\beta]=0,[\alpha] \cup[\beta]=0^{\prime}$.

Proof. Choose $a \in O^{*}-O$ such that $(\exists \beta)(\exists v)(\forall x)\left(H(\bar{\beta}(x), \bar{\nu}(x), a) \wedge \bar{\beta}(1, x)_{0}=1\right)$. By Theorem 2.12 we can choose a $\beta$ with this property, and, in addition, $\alpha \cap_{H} \beta$ $\subseteq H A, \beta<{ }_{H} O^{\alpha}={ }_{H} O$ (since $\alpha<_{H} O$ ). Since $a \in O^{*}-O$ and $\beta$ is a hierarchy for $a$ with initial function identically one, all hyperarithmetic sets are recursive in $\beta$. Hence $H A \subseteq \alpha \cap_{R} \beta \subseteq \alpha \cap_{H} \beta \subseteq H A$, i.e., $\alpha \cap_{R} \beta=H A$. But then it follows that $O \leqq_{H} \alpha \cup \beta$ since by Spector's Theorem [15] we have for suitably chosen recursive $S: n \in O \leftrightarrow(\exists \delta)_{H A}(\forall x) S(n, \delta(x)) \leftrightarrow(\exists m)(\exists k)\left(\{m\}^{\alpha},\{k\}^{\beta}\right)$ are total and $\{m\}^{\alpha} \equiv\{k\}^{\beta}$ $\wedge(\forall x) S\left(n,\{\bar{m}\}^{\alpha}(x)\right)$. This shows $O$ is arithmetic in $\alpha \cup \beta$. Since $\alpha, \beta<{ }_{H} O$, we must have $O={ }_{H} \alpha \cup \beta$. Hence the hyperdegree of the function $\beta$ has the properties required by the theorem.

This corollary answers a question of Sacks and Thomason.

We conclude with two disparate remarks. First, all results in this paper relativize to pseudo- $\alpha$-well-orderings, where $\alpha$ is an arbitrary function. In particular, by relativization we obtain for each hyperdegree $\alpha$ the existence of a densely ordered set of hyperdegrees whose first element is $\alpha$ and which has the maximality property of Corollary 2.9 so that $\alpha$ is the greatest lower bound of the hyperdegrees of this set.

Second, the main open question on hierarchies is whether $(\forall a)\left(a \in O^{*}\right.$ $\rightarrow(\exists \alpha) H(\alpha, a))$. By our remark following Theorem 2.5 this result cannot be proved solely by means of the $\Sigma_{1}^{1}$ axiom of dependent choices even for $O$. Hence, a solution to this question would probably require an interesting new method $\left({ }^{4}\right)$.

\section{BIBLIOGRAPHY}

1. S. Feferman, An w-model for the hyperarithmetic comprehension axiom in which the $\Sigma_{1}^{1}$ axiom of choice fails, Proc. Internat. Congr. Math., Moscow, 1966.

2. - Classification of recursive functions by means of hierarchies, Trans. Amer. Math. Soc. 104 (1962), 101-122.

3. — Constructive pseudo-well-orderings, Notices Amer. Math. Soc. 9 (1962), 136.

$\left.{ }^{3}\right)$ The second part of the conclusion of Theorem 2.12 has since been obtained by $R$. $O$. Gandy by a direct argument not involving the use of nonstandard hierarchies.

(4) R. M. Friedman has answered this question negatively. 
4. - Some applications of the notions of forcing and generic sets, Fund. Math. 16 (1965), 383-390.

5. S. Feferman and C. Spector, Incompleteness along paths in progressions of theories, J. Symbolic Logic 27 (1962), 383-390.

6. R. O. Gandy, On a problem of Kleene's, Bull. Amer. Math. Soc. 66 (1960), 501-502.

7. _-, Proof of Mostowski's conjecture, Bull. Acad. Polon. Sci. Ser. Sci. Math. Astronom. Phys. 8 (1960), 571-575.

8. J. Harrison, Further results on $O^{*}$, Notices Amer. Math. Soc. 12 (1965), 604.

9. - Extensions of the hyperarithmetic hierarchy, Notices Amer. Math. Soc. 12 (1965), 806.

10. - Pairs of hyperdegrees without a greatest lower bound, Notices Amer. Math. Soc. 13 (1965), 623.

11. - Some applications of recursive pseudo-well-orderings, Ph.D. Thesis, Stanford Univ., Stanford, Calif., 1966.

12. S. C. Kleene, On the forms of predicates in the theory of constructive ordinals. II, Amer. J. Math. 77 (1954), 379-407.

13. G. Kreisel, Some axiomatic results on second order arithmetic, Seminar notes, Stanford Univ., Stanford, Calif., 1963.

14. - The axiom of choice and the class of hyperarithmetic functions, Nederl. Acad. Wetensch. Proc. Ser. A $65=$ Indag. Math. 24 (1962), 307-319.

15. C. Spector, Hyperarithmetic quantifiers, Fund. Math. 48 (1960), 313-320.

16. - Measure theoretic construction of incomparable hyperdegrees, J. Symbolic Logic 23 (1958), 280-288.

17. —-, Recursive well-orderings, J. Symbolic Logic 20 (1955), 151-163.

18. S. K. Thomason, The forcing method and the upper semilattice of hyperdegrees, Ph.D. Thesis, Cornell Univ., Ithaca, N. Y., 1965.

STANFORd UNIVERSITY, Stanford, California 